\title{
Infestazione spuria da Dicrocoelium dendriticum
}

\section{Maria Letizia D'Annibale}

Sezione di Microbiologia e Parassitologia Clinica, Ospedale "R. Silvestrini”, Perugia

\section{DESCRIZIONE DEL PRESUNTO CASO}

Un soggetto di sesso maschile di 74 anni, autoctono, viene invitato ad eseguire un esame coproparassitologico in quanto affetto da blandi disturbi intestinali accompagnati da prurito cutaneo con lesioni escoriative, con una eosinofilia periferica del $7 \%$.

Alla consegna, in ambulatorio, dei 3 campioni fecali, il soggetto riferisce anche una già documentata allergia cutanea agli alcoli della lanolina (usati come additivi di svariati medicamenti topici, creme, saponi, lozioni, cosmetici e analoghi), da cui la verosimile patologia cutanea.

Il soggetto riferisce peraltro anche problemi cardiaci con ipertensione arteriosa per i quali è in cura specifica da tempo.

Non riferisce però fattori di rischio specifico per una potenziale parassitosi intestinale.

\section{INDAGINI PARASSITOLOGICHE}

Dei 3 campioni fecali ne viene fatto un adeguato pool, che viene successivamente esaminato come esame coproparassitologico standard (5).

Vengono così condotte osservazioni microscopiche dirette (in soluzione fisiologica e in soluzione di Dobell), un arricchimento per concentrazione formolo-etilacetato (FEA: ConTrate, Oxoid), una colorazione di Giemsa (1).

Le osservazioni microscopiche (dirette, $40 \times 10$, e dopo colorazione di Giemsa, $100 \times 10$, in immersione) mettono in evidenza la presenza di strutture vacuolari di Blastocystis hominis, protozoo tipicamente saprofita opportunista (2).

L'osservazione microscopica dopo FEA mette in evidenza, invece, un discreto numero di uova di Dicrocoelium dendriticum, di colorazione pleomorfa: bruno chiare- grigie e bruno scure (Figure I e II). Le dimensioni delle uova, opercolate, sono più o meno quelle usuali: 40-45 x 20-26 $\mu \mathrm{m}$.

Tali uova vengono interpretate come di transito passivo, in seguito a verosimile ingestione di alimenti animali (fegato) contenenti tali uova.

Peraltro, la inusuale numerosità delle uova mi invita a contattare ulteriormente il soggetto per meglio comprendere il caso e per poi procedere ad un altro esame coproparassitologico per verificare la eliminazione delle medesime uova.

Nelle infestazioni spurie, nell'arco di 3 giorni le uova, accidentalmente ingerite, vengono solitamente del tutto eliminate (4).

\section{ASPETTI BIOLOGICI}

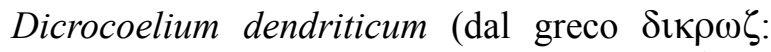
doppio, $\chi 0 \imath \lambda \iota \alpha$ : cavità del ventre; $\delta \varepsilon \nu \tau \rho \imath \tau \imath \xi o v:$ ramificato) è cosmopolita, frequente in Italia, presente in ovini e bovini, nei quali può essere causa di gravi danni (7). È infatti ospite definito di questi mammiferi, laddove primo ospite intermedio sono molluschi vari e secondo ospite intermedio sono le formiche (Formica fusca ed altre). L'uovo, eliminato dagli animali infestati, resiste a lungo all'essicamento, per poi venire ingerito dal mollusco idoneo, nel quale si sviluppa a sporocisti e poi a cercarie; queste ultime sono ingerite dalla formica, nella quale si sviluppano a metacercarie.

L'ospite definitivo si infesta ingerendo le formiche parassitate, le quali, per le lesioni provocate dalle metacercarie al loro sistema nervoso, perdono la capacità di muoversi e restano bloccate sulle foglie dell'erba che l'erbivoro, appunto, ingerisce.

Le metacercarie, nel mammifero, dall'intestino raggiungono poi il fegato. Da quanto detto se ne evince la estrema rarità di una infestazione vera nell'uomo $(3,6,7)$.

\section{CONSIDERAZIONI CONCLUSIVE}

Il soggetto in questione venne così invitato a ripresentarsi al nostro ambulatorio.

Al soggetto venne spiegata la situazione e lo stesso riferì che, in verità, riposando sotto gli alberi del giardino di casa, in ambiente di campagna, molte formiche gli cadevano addosso, e non escluse la possibilità di una loro ingestione.

Sotto nostro invito raccolse svariate formiche e le portò alla nostra osservazione.

Tali formiche erano peraltro vive e vitali, per cui si escluse una loro infezione. Ma l'informazione più rilevante a definire il "falso" caso fu la confessione di avere consumato, due settimane prima ad una sagra paesana, "coratella d'agnello" (che contiene anche fegato ovino). Tre successivi campioni fecali analizzati diedero, come atteso, esito negativo.

Riportiamo questa infestazione spuria da $D$. dendriticum, tutt'altro che infrequente, esclusivamente per richiamare all'attenzione i percorsi diagnostici adeguati e per la importanza di comprendere sempre fino a fondo qualsivoglia situazione clinica particolare, vera o falsa che essa sia o possa essere. 


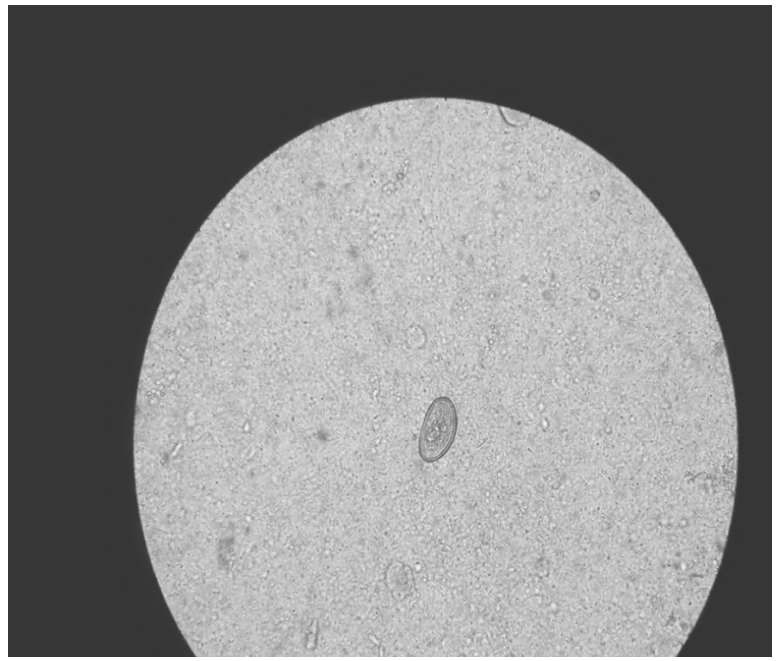

Figura I. Uova "chiare" di Dicrocoelium dendriticum

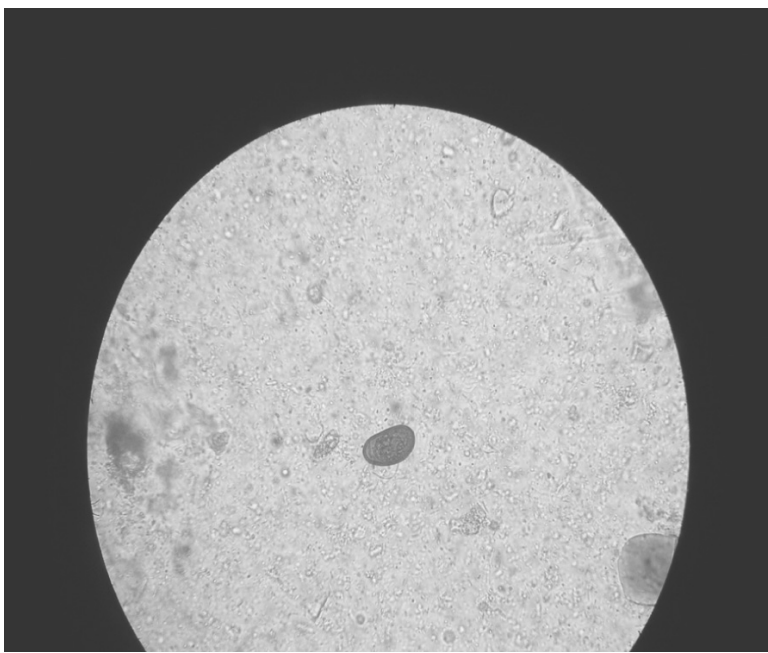

Figura II. Uova “scure” di Dicrocoelium dendriticum

\section{BIBLIOGRAFIA}

1. Bernieri F, Crotti D, Galli D, Raglio A. Manuale illustrato di diagnostica parassitologica. Edizioni Selecta Medica, Pavia, 2001

2. De Carneri I. Blastocystis hominis. In : Parassitologia generale e umana. Casa Editrice Ambrosiana, Milano, XI Edizione, 1992; 177

3. Duchaçek L, Lamka J. Dicrocoeliosi-the Present State of Knowledge with Respect to Wildlife Species. Acta Vet. BRNO 2003, 72: 613-626

4. Garcia LS. Less Common Liver Flukes. In: Diagnostic Medical Parasitology. ASM Press, Washington, D. C., IV Edition, 2001; 436-438

5. Garcia LS. Practical Guide to Diagnostic Parasitology. ASM Press, Washington, D. C., 1999

6. Lloyd S, Soulsby EJL. Other Trematode Infections. In: Zoonoses, Edited by S. R. Palmer, Lord Soulsby and D. I. H. Simpson, Oxford University Press, New York, 1998; 732-746

7. Pampiglione - Canestri Trotti. Dicrocoelium dendriticum. In: Guida allo studio della Parassitologia. Società Editrice Esculapio, Bologna, III Edizione, $1999 ; 145-147$

\section{Maria Letizia D'Annibale}

Sezione di Microbiologia e Parassitologia Clinica, Azienda Ospedaliera di Perugia, Ospedale "R. Silvestrini", via G. Dottori 1, 06156 S. Andrea delle Fratte, Perugia Telefono 075 5782218; Telefax 0755782404 E-mail: analisi.cliniche@ospedale.perugia.it m.letiziadannibale@tin.it 